\title{
Fatores prognósticos e sobrevida em recém-nascidos com hérnia diafragmática congênita
}

\section{Prognostic factors and survival in neonates with congenital diaphragmatic hernia}

\author{
Luis R. Longo dos Santos ${ }^{1}$, João G. Maksoud-Filho² ${ }^{2}$ Uenis Tannuri ${ }^{3}$, \\ Wagner C. Andrade ${ }^{4}$, João G. Maksoud ${ }^{5}$
}

\section{Resumo}

Objetivo: analisar a importância de fatores prognósticos para sobrevida, no período neonatal, de pacientes com hérnia diafragmática congênita (HDC) tratados no Hospital das Clínicas da FMUSP e comparar os resultados com os da literatura. Com isso, permitir a atualização do aconselhamento familiar e orientar mudanças de conduta em nosso serviço.

Método: estudo retrospectivo de 27 casos consecutivos de recémnascidos com HDC, admitidos no serviço de cirurgia pediátrica do Instituto da Criança do HCFMUSP entre abril de 1991 e janeiro de 2002, e comparação estatística com dados de meta-análise da literatura.

Resultados: dos 27 pacientes, 12 nasceram na instituição, e 15 foram transferidos após o nascimento. Doze (44\%) tiveram diagnóstico pré-natal, e 85\% (23/27) eram nascidos de termo. A maioria apresentou desconforto respiratório precoce e necessitou intubação em sala de parto. Seis crianças $(22 \%)$ reuniam critérios para indicação de ECMO. Vinte pacientes $(74 \%)$ foram submetidos à correção cirúrgica, e sete (26\%) evoluíram a óbito sem conseguir estabilização clínica mínima para a cirurgia (cinco destes apresentavam critérios para indicação de ECMO). A mortalidade pós-operatória foi de $25 \%$ (5/20). A sobrevida global no período neonatal foi de 56\% (15/27). A sobrevida dos nascidos em nosso hospital foi de 33\% (4/12), e dos casos externos foi de $73 \%$ (11/15). Desconforto respiratório grave, indicação precoce de ventilação mecânica e hipoxemia grave ( $\mathrm{pO}_{2}$ pós-ductal $<100 \mathrm{mmHg}$ apesar de terapêutica máxima) foram fatores de mau prognóstico, estatisticamente significativos.

Conclusão: nossa elevada mortalidade de recém-nascidos com HDC é estatisticamente semelhante à descrita na literatura internacional. No grupo de pacientes não responsivos ao tratamento padrão disponível, a utilização de ECMO poderia reduzir a mortalidade. A repercussão desta estratégia terapêutica na sobrevida global depende de outros fatores não analisados neste estudo. $\mathrm{O}$ aconselhamento dos familiares de pacientes com HDC, em nosso serviço, pode seguir o mesmo padrão do referido na literatura mundial.

JPediatr (Rio J) 2003;79(1):81-6: hérnia diafragmática congênita, ECMO, insuficiência respiratória no recém-nascido.

\begin{abstract}
Objective: to evaluate the prognostic factors importance to survival in neonatal period of newborns with congenital diaphragmatic hernia treated at Hospital das Clínicas, School of Medicine of Universidade de São Paulo, and to compare the outcome with data published in medical literature. So that the results of this study might allow updating family counseling and guiding changes in clinical management of our department.

Methods: retrospective study of 27 consecutive newborns with congenital diaphragmatic hernia admitted to the Pediatric Surgery Department of Instituto da Criança, School of Medicine of Universidade de São Paulo, from April 1991 to January 2002, and statistical comparison with medical literature metanalysis data.

Results: of 27 patients, 15 were born at our institution and 12 were admitted by transference after birth. Twelve (44\%) have had congenital diaphragmatic hernia diagnosed prenatally and $23(85 \%)$ were full-term newborns. Most patients presented early respiratory distress and needed intubation at delivery room. Six newborns presented criteria for indication of extracorporeal membrane oxygenation. Twenty patients $(74 \%)$ were submitted to operative repair and seven $(26 \%)$ died without the minimal clinical stabilization necessary for surgical procedure (five of these patients reached criteria for indication of extracorporeal membrane oxygenation). The postoperative mortality was $25 \%(5 / 20)$. The overall survival of neonatal period was $56 \%(15 / 27)$. The survival of patients that were born at our hospital was 33\% (4/12), and the survival of the newborns admitted by transference was $73 \%$ (11/15). Severe respiratory distress, early indication to mechanical ventilation and severe hypoxemia (post-ductal $\mathrm{pO}_{2}<100 \mathrm{mmHg}$ despite all efforts) were identified as predictors of bad outcome with statistical significance.
\end{abstract}

Conclusion: our high mortality rate of newborns with congenital diaphragmatic hernia is statistically similar to that described in international publications. In the group of non-responsive patients to standard treatment available, the use of extracorporeal membrane oxygenation should be able to reduce mortality. The impact of this therapeutical strategy in the overall survival depends on other factors that were not analyzed in the present study. Family counseling of patients' parents on congenital diaphragmatic hernia in our department may follow the same patterns referred in world medical literature.

JPediatr (Rio J) 2003;79(1):81-6: congenital diaphragmatic hernia, extracorporeal membrane oxygenation, newborn respiratory failure.

1. Médico Preceptor da Disciplina de Cirurgia Pediátrica do HCFMUSP.

2. Doutor em Cirurgia pela Faculdade de Medicina da Universidade de São Paulo. (FMUSP).

3. Professor Associado da Disciplina de Cirurgia Pediátrica da FMUSP.

4. Residente de Cirurgia Pediátrica do ICr-HCFMUSP.

5. Professor Titular da Disciplina de Cirurgia Pediátrica da FMUSP.

Artigo submetido em 03.09.02, aceito em 06.11.02. 
$\mathrm{O}$ tratamento de recém-nascidos $(\mathrm{RN})$ com hérnia diafragmática congênita (HDC) continua sendo um desafio para cirurgiões pediátricos e neonatologistas.

Nas últimas décadas, avanços na terapia da hipertensão pulmonar primária (HPP) do recém-nascido, tais como ventilação suave com hipercapnia permissiva ${ }^{1,2}$, ventilação por oscilação de alta freqüência $(\mathrm{VAF})^{3}$, uso de óxido nítrico $(\mathrm{ON})$ como vasodilatador pulmonar seletivo ${ }^{4} \mathrm{e}$ oxigenação extracorpórea por membrana $(\mathrm{ECMO})^{5,6}$ foram incorporados ao tratamento de RN portadores de HDC. Paralelamente, a possibilidade de diagnóstico ultra-sonográfico pré-natal e as tentativas de intervenção intra-útero abriram novas perspectivas de sucesso ${ }^{7-9}$. Apesar de relatos iniciais apontando redução na mortalidade, algumas análises retrospectivas não confirmaram um impacto significativo na sobrevida global ${ }^{10-12}$.

O binômio hipoplasia-hipertensão pulmonar permanece como principal fator de mortalidade ${ }^{13}$. Vários fatores prognósticos, pré e pós-natais, foram descritos para definir a gravidade da doença e as chances individuais de sobrevida, objetivando orientação de condutas médicas e aconselhamento familiar ${ }^{14-20}$. Embora nenhum desses parâmetros possa ser considerado isoladamente, alguns continuam mundialmente aceitos e utilizados para avaliação comparativa da qualidade dos cuidados oferecidos a esses recémnascidos $^{21}$.

Recentemente, Beresford e Shaw apresentaram metaanálise baseada em 35 publicações relativas a evolução de RNs ou de fetos com diagnóstico de HDC ${ }^{22}$. Nesta avaliação, foram comparados dados de mortalidade, correlacionando-os ao diagnóstico e ao tratamento realizados. Os resultados estão resumidos na Tabela 1 .

O objetivo de nosso trabalho é analisar a importância de fatores prognósticos na sobrevida no período neonatal de pacientes com HDC tratados no Hospital das Clínicas da Faculdade de Medicina da USP (HCFMUSP), e comparar os resultados com os da literatura, permitindo, com isso, gerar dados atualizados para a orientação familiar e para mudanças de conduta em nosso serviço.

\section{Método}

Análise retrospectiva de prontuários de 27 recém-nascidos (RN) com HDC, tratados pelo serviço de cirurgia pediátrica do ICr-HCFMUSP, entre abril de 1991 e janeiro de 2002.

O tratamento empregado em cada caso, conforme a gravidade, incluiu: (1) suporte hidroeletrolítico e nutricional por via parenteral; (2) controle hemodinâmico e gasométrico, a partir de cateterização de artéria umbilical, tibial ou radial; (3) suporte respiratório através de oxigenoterapia suplementar, ventilação mecânica convencional com hipercapnia permissiva, ou ventilação por oscilação de alta freqüência; (4) uso de surfactante exógeno, sedação, drogas vasoativas e óxido nítrico inalatório; (5) indicação cirúrgica após estabilização clínica (cirurgia retardada) ${ }^{23-26}$.

Os fatores prognósticos analisados foram: desconforto respiratório ao nascimento (escore de Apgar $\leq 6$ no 5으 minuto de vida), necessidade de intubação precoce $(<6$ horas de vida), hipoxemia grave mantida $\left(\mathrm{pO}_{2}\right.$ pós-ductal inferior a $100 \mathrm{mmHg}$ apesar de terapêutica máxima), associação de malformações somáticas graves, hipertensão pulmonar grave (ecocardiograma com gradiente $>40 \mathrm{mmHg}$ ou repercussão em VD) e indicação potencial de ECMO (definida como $\mathrm{pO}_{2}<50 \mathrm{mmHg}$ por mais de 4 horas e ausência de resposta à terapêutica convencional disponível em RN com peso maior que $2.000 \mathrm{~g}$, sem hemorragia de SNC e sem malformações cardíacas ou outras incompatíveis com a vida) ${ }^{27}$.

Os dados referentes à mortalidade e aos fatores prognósticos foram analisados e comparados com aqueles apresentados por Beresford et al., utilizando o teste do quiquadrado com correção de Yates, ou teste exato de Fisher uni ou bicaudal, com significância para valores de $\mathrm{p}<0,05$.

A concepção e a realização deste trabalho foram aprovadas pela Comissão de Ética em Pesquisa de nossa instituição.

\section{Resultados}

Dentre os 27 pacientes, 12 nasceram em nossa instituição e 15 foram transferidos com intervalo médio de 3,2 dias de vida ( 15 horas-9 dias). Doze (44\%) tiveram diagnóstico pré-natal, sendo 11 destes nascidos no HCFMUSP. O peso médio de nascimento foi de $2.805 \mathrm{~g}(1.107-4.420 \mathrm{~g})$, com $85 \%$ (23/27) nascidos de termo.

Foram diagnosticadas malformações congênitas complexas em 8 casos (30\%), sendo três (11\%) malformações cardíacas.

Em relação a fatores prognósticos, 56\% (15/27) apresentaram escore de Apgar $\leq 6$ no 5o minuto de vida, e 59\% (16/27) foram intubados precocemente (maioria em sala de parto). A hipertensão pulmonar (HP) foi avaliada no préoperatório através de ecocardiograma em 70\% (19/27) dos pacientes. Destes, metade apresentava HP grave.

Seis crianças apresentavam critérios de indicação de ECMO, conforme definidos acima, porém o método não estava disponível. Apenas uma destas sobreviveu.

Vinte pacientes $(74 \%)$ foram submetidos à correção cirúrgica, e sete (26\%) evoluíram a óbito sem alcançar condições clínicas mínimas para cirurgia. Destes que morreram na fase de preparo pré-operatório, cinco seriam candidatos a ECMO, e em dois o método estaria contraindicado, devido a baixo peso e cardiopatia grave. A mortalidade pós-operatória foi de $25 \%(5 / 20)$. Não ocorreram óbitos intra-operatórios. 
Tabela 1 - Mortalidade calculada dependendo do estágio alcançado pelos pacientes com diagnóstico de HDC, metaanálise de Beresford et al.

\begin{tabular}{lccccc}
\hline $\begin{array}{l}\text { Dados / } \\
\text { estudo estatístico }\end{array}$ & $\begin{array}{c}\text { Diagnóstico } \\
\text { pré-natal }\end{array}$ & $\begin{array}{c}\text { Nascidos } \\
\text { vivos }\end{array}$ & $\begin{array}{c}\text { RN } \\
\text { operados }\end{array}$ & $\begin{array}{c}\text { Óbito } \\
\text { fetal }\end{array}$ & $\begin{array}{c}\text { Óbito } \\
\text { pré-peratório }\end{array}$ \\
\hline Mortalidade mediana (IQR) * & $58 \%(43-65)$ & $48 \%(35-55)$ & $33 \%(18-54)$ & $10 \%(7-25)$ & $27 \%(14-43)$ \\
Mortalidade média (IC 95\%) & $56 \%(46-66)$ & $47 \%(40-54)$ & $36 \%(27-45)$ & $14 \%(4-24)$ & $30 \%(20-40)$ \\
$\mathrm{N}^{\circ}$ trabalhos analisados & 9 & 20 & 27 & 6 & 15 \\
$\mathrm{~N}^{\mathbf{o}}$ pacientes & 285 & 844 & 794 & 212 & 538 \\
$\mathrm{~N}^{\mathbf{o}}$ óbitos & 164 & 372 & 269 & 29 & 139 \\
\hline
\end{tabular}

* variação interquartil † intervalo de confiança de 95\%

A mortalidade global no período neonatal foi de $44 \%$ (12/27). A mortalidade dos RN externos foi de 27\% (4/15) e dos nascidos no HCFMUSP foi de 67\% (8/12).

A mortalidade em diferentes subgrupos, comparada com aquela descrita por Beresford e Shaw, é apresentada na Tabela 2. A análise dos fatores prognósticos em nossa casuística está discriminada na Tabela 3.

\section{Discussão}

O grupo de recém-nascidos com HDC, tratados em nossa instituição no período analisado, é bastante heterogêneo, incluindo desde crianças com desconforto respiratório moderado e sem outras anomalias, até aquelas com grave hipoplasia pulmonar e malformações complexas associadas. Estas diferenças explicam, em parte, a discrepância entre a mortalidade dos casos acompanhados desde o prénatal e nascidos em nossa instituição, e dos casos diagnosticados e transferidos após o nascimento. No primeiro grupo, concentra-se a maioria dos RNs em estado grave e com pior prognóstico. A menor mortalidade dos casos transferidos após o nascimento e sem diagnóstico pré-natal pode ser atribuída a viés de seleção, pois muitos dos casos graves atendidos inicialmente em serviços de neonatologia com menores recursos terapêuticos evoluem a óbito antes que seja possível a transferência para serviços especializados e não são, portanto, incluídos nas estatísticas ${ }^{28}$.

Boloker et al., analisando 120 casos consecutivos de HDC, tratados em um único centro e com terapêutica padronizada, encontraram também diferenças entre o grupo de RN nascidos na instituição e os transferidos após o nascimento, observando, no segundo, menor incidência de malformações graves e menor mortalidade ${ }^{29}$. Concluíram que estas diferenças estavam relacionadas a uma pré-seleção e que, excluindo-se os casos com malformações somáticas e/ou pulmonares incompatíveis com a vida e os casos com complicações neurológicas, é possível atingir sobrevida superior a $80 \%$ através do tratamento com ventilação suave e cirurgia retardada, evitando a mortalidade relacionada a iatrogenias.

Tabela 2 - Comparação de mortalidade por subgrupos entre casuística do ICr-HCFMUSP e meta-análise de Beresford et al.

\begin{tabular}{lccl}
\hline Mortalidade & ICr-HCFMUSP & Beresford et al. \\
\hline 1. Crianças nascidas vivas & $44 \%(12 / 27)$ & $48 \%$ & $\mathrm{p}=0,87$ \\
2. Diagnóstico pré-natal & $67 \%(8 / 12)$ & $58 \%$ & $\mathrm{p}=0,74$ \\
3. RN com indicação de ECMO & $83 \%(5 / 6)$ & $44 \%$ & $\mathrm{p}=0,06$ unicaudal \\
4. Crianças em preparo pré-operatório & $26 \%(7 / 27)$ & $27 \%$ & $\mathrm{p}=0,82$ \\
5. Crianças operadas & $25 \%(5 / 20)$ & $33 \%$ & $\mathrm{p}=0,55$ \\
\hline
\end{tabular}


Tabela 3 - Correlação entre fatores prognósticos analisados e a mortalidade em nosso meio

\begin{tabular}{lccc}
\hline Fator prognóstico & $\begin{array}{c}\text { Mortalidade na } \\
\text { presença do fator }\end{array}$ & $\begin{array}{c}\text { Mortalidade na } \\
\text { ausência do fator }\end{array}$ \\
\hline 1. Intubação $<6$ horas de vida & $69 \%$ & $9 \%$ & $\mathrm{p}=0,005^{*}$ \\
2. Impossibilidade de $\mathrm{pO}_{2}>100$ & $100 \%$ & $32 \%$ & $\mathrm{p}=0,01^{*}$ \\
3. Apgar 5 $5^{\circ}$ minuto $\leq 6$ & $67 \%$ & $17 \%$ & $\mathrm{p}=0,02^{*}$ \\
4. HP grave ao ecocardiograma & $67 \%$ & $35 \%$ & $\mathrm{p}=0,12$ \\
5. Diagnóstico pré-natal & $67 \%$ & $27 \%$ & $\mathrm{p}=0,09$ \\
6. Malformações graves associadas & $62 \%$ & $39 \%$ & $\mathrm{p}=0,40$ \\
\hline
\end{tabular}

*Valores estatisticamente significativos.

Comparando a mortalidade em nossa casuística e a sumarizada pela meta-análise publicada por Beresford e Shaw, não encontramos diferença estatisticamente significativa em nenhum dos subgrupos analisados. A mortalidade em nossa instituição é, inclusive, na maioria dos subgrupos, numericamente semelhante à média encontrada na literatura.

Entretanto, entre as crianças com critérios para indicação de ECMO, nossa mortalidade foi quase o dobro da referida na literatura ( $83 \%$ versus $44 \%, \mathrm{p}=0,06$ unicaudal). Em função do tamanho da amostra, não foi possível estabelecer significância estatística nesta casuística. Porém, o próprio intervalo de confiança, de $95 \%$, estabelecido para a média de mortalidade dos pacientes com indicação de ECMO na meta-análise de Beresford (34-50\%) está bastante afastado da nossa mortalidade neste subgrupo. Das sete crianças que evoluíram a óbito antes de atingir condições clínicas mínimas para a cirurgia, cinco tinham critérios para indicação de ECMO. Estes dados sugerem que a efetiva utilização de ECMO em nosso serviço poderia reduzir a mortalidade nesse subgrupo. A repercussão na mortalidade global dependeria, entretanto, do número de crianças admitidas com indicação de ECMO e de nossos resultados efetivos com o método.

A definição de fatores prognósticos confiáveis é uma preocupação constante dos centros que tratam pacientes com HDC. Com base nesses fatores, seria possível diferenciar as crianças que se beneficiariam efetivamente de terapêuticas agressivas e dispendiosas daquelas em que esse tratamento serviria apenas para prolongar o sofrimento do paciente e de seus familiares.
Vários fatores prognósticos foram descritos por diversos centros, tais como anoxia perinatal e precocidade do início dos sintomas, índices ventilatórios, medidas de complacência pulmonar, ocorrência de pneumotórax decorrente de ventilação agressiva, capacidade de atingir $\mathrm{pO}_{2}>100$ $\mathrm{mmHg}$, grau de hipertensão pulmonar, precocidade do diagnóstico antenatal, tamanho do defeito diafragmático, herniação do fígado para o tórax, associação de cardiopatia ou outras malformações complexas ${ }^{30}$. Estes parâmetros relacionam-se com a gravidade da hipoplasia pulmonare da hipertensão pulmonar. Assim, RNs com sintomas precoces e intensos, e não-responsivos à terapêutica, apresentariam graves malformações pulmonares e outras de difícil reversão agudamente, enquanto em outros a hipertensão pulmonar pode ser manejada com diferentes estratégias, quebrando-se o círculo vicioso de HP - hipercapnia e hipoxemia acidose - HP agravada.

Nenhum fator isoladamente se mostrou ideal para prever a sobrevida em todas as instituições. Isso decorre tanto da variabilidade dos tratamentos empregados em cada centro como da extrema instabilidade clínica desses RNs, que podem apresentar deterioração clínica súbita devido à piora da hipertensão pulmonar.

Dos fatores prognósticos desfavoráveis classicamente referidos, obteve-se correlação estatística, em nossa casuística, para necessidade de intubação precoce, escore de Apgar $\leq 6$ no $5^{\circ}$ minuto de vida, e impossibilidade de atingir $\mathrm{pO}_{2}$ maior que $100 \mathrm{mmHg}$ apesar da terapêutica máxima. Os demais fatores analisados, embora numericamente sugerissem relevância, não foram estatisticamente significativos devido ao tamanho da amostra analisada. 
Concluímos que a mortalidade de recém-nascidos com diagnóstico de HDC permanece elevada em nosso serviço, porém em níveis semelhantes à média dos publicados na literatura internacional. No grupo de pacientes não-responsivos ao tratamento padrão disponível, a introdução da ECMO poderia possibilitar redução da mortalidade. A repercussão desta medida na sobrevida global depende de outros fatores não analisados neste estudo. A orientação dos familiares de pacientes com HDC em nosso serviço pode seguir o mesmo padrão do referido na literatura internacional.

\section{Referências bibliográficas}

1. Hansen J, James S, Burrington JD. The decreasing incidence of pneumothorax and improving survival of infant with congenital diaphragmatic hernia. J Pediatr Surg 1984;19:385-8.

2. Wung JT, James LS, Kilchevsky E, James E. Management of infants with severe respiratory failure and persistence of the fetal circulation, without hyperventilation. Pediatrics 1985;76:488-94.

3. Clark RH, Bradley LTC, Yoder A. Prospective, randomised comparison of high-frequency oscillation and conventional ventilation in candidates for extracorporeal membrane oxygenation. J Pediatr 1994;124:447-54.

4. Neonatal Inhaled Nitric Oxide Study Group. Inhaled nitric oxide and hypoxic respiratory failure in infants with congenital diaphragmatic hernia. Pediatrics 1997;99:838-45.

5. Walker LK. Use of extracorporeal membrane oxygenation for preoperative stabilization of congenital diaphragmatic hernia. Crit Care Med 1993;21 Suppl 9:379-80.

6. Ssemakula N, Stewart DL, Goldsmith LJ, Cook LK, Bond SJ. Survival of patients with congenital diaphragmatic hernia during the ECMO era: an 11-year experience. J Pediatr Surg 1997;32:1683-9.

7. Steinhorn RH, Kriesmer PJ, Green TP. Congenital diaphragmatic hernia in Minnesota. Impact of antenatal diagnosis on survival. Arch Ped Adol Med 1994;148:626-31.

8. Dommergues M, Louis-Sylvestre C, Mandelbrot L, Oury JF, Herlicoviez M, Body G, et al. Congenital diaphragmatic hernia: can prenatal ultrasonography predict outcome? Am J Obstet Gynecol 1996;174:1377-81.

9. Harrison MR, Adzick NS, Bullard KM, Farrell JA, Howell LJ, Rosen MA, et al. Correction of congenital diaphragmatic hernia in uterus VII: a prospective trial. J Pediatr Surg 1997;32:1637-42.

10. The Congenital Diaphragmatic Hernia Study Group. Does extracorporeal membrane oxygenation improve survival in neonates with congenital diaphragmatic hernia? J Pediatr Surg 1999;34:720-25.

11. Wilson JW, Lund DP, Lillehei CW. Delayed repair and preoperative ECMO does not improve survival in high-risk congenital diaphragmatic hernia. J Pediatr Surg 1992;27:368-72.
12. West KW, Bengston K, Rescorla FJ. Delayed repair and ECMO improves survival in congenital diaphragmatic hernia. Ann Surg 1992;216:454-60.

13. Bohn D, Tamura M, Perrin D. Ventilatory predictors of pulmonary hypoplasia in congenital diaphragmatic hernia confirmed by morphometry. J Pediatr 1987;111:423-31.

14. Sreenan C, Etches P, Osiovich H. The western Canadian experience with congenital diaphragmatic hernia: perinatal factors predictive of extracorporeal membrane oxygenation and death. Pediatr Surg Int 2001;17:196-200.

15. Wilson JM, Lund DP, Lillehei CW, Vacanti JP. Congenital diaphragmatic hernia: predictors of severity in the ECMO era. J Pediatr Surg 1991;26:1028-33.

16. Suda K, Bigras JL, Bohn D, Hornberger LK, McCrindle BW. Echocardiographic predictors of outcome in newborns with congenital diaphragmatic hernia. Pediatrics 2000;105:1106-9.

17. Kaiser JR, Rosenfeld CR. A population-based study of congenital diaphragmatic hernia: impact of associated anomalies and preoperative blood gases on survival. J Pediatr Surg 1999;34:1196-202.

18. Fauza DO, Wilson JM. Congenital diaphragmatic hernia and associated anomalies: their incidence, identification, and impact on prognosis. J Pediatr Surg 1994;29:1113-7.

19. Bohn D. Ventilatory and blood gas parameters in predicting survival in congenital diaphragmatic hernia. Pediatr Surg Int 1987;2:336-40.

20. Germain JF, Farnoux C, Pinquier D, Cortez A, Hartmann JF, Sibony O, et al. Can blood gas values predict pulmonary hypoplasia in antenatally diagnosed congenital diaphragmatic hernia? J Pediatr Surg 1996;31:1634-9.

21. Breaux CWJ, Rouse TM, Cain WS, Georgeson KE. Congenital diaphragmatic hernia in an era of' delayed repair after medical and/or extracorporeal membrane oxygenation stabilization: a prognostic and management classification. J Pediatr Surg 1992;27:1192-6.

22. Beresford MW, Shaw NJ. Outcome of congenital diaphragmatic hernia. Pediatr Pulmonol 2000;30:249-56.

23. Sakai H, Tamura M, Hosokawa Y. Effect of surgical repair on respiratory mechanics in congenital diaphragmatic hernia. $\mathrm{J}$ Pediatr 1987;111:432-8.

24. Nakayama DK, Motoyama EK, Tagge EM. Effect of preoperative stabilization on respiratory system compliance and outcome in newborn infants with congenital diaphragmatic hernia. J Pediatr 1991;118:793-9.

25. Nio M, Haase G, Kennaugh J, Bui K, Atkinson JB. A prospective randomised trial of delayed vs. immediate repair of congenital diaphragmatic hernia. J Pediatr Surg 1994;29:618-21.

26. de la Hunt MN, Madden N, Scott JES, Matthews JNS, Beck J, Sadler C. Is delayed surgery really better for congenital diaphragmatic hernia? A prospective randomised clinical trial. $\mathrm{J}$ Pediatr Surg 1996;31:1554-6.

27. Rosenberg EM, Seguin JH. Selection criteria for use of ECLS in neonates. In: Zwischenberger JB, Bartlett RH. ECMO. Extracorporeal Cardiopulmonary Support in Critical Care. Extracorporeal Life Support Organization; 1995. p. 261-74.

28. Jaffray B, MacKinlay GA. Real and apparent mortality from congenital diaphragmatic hernia. Br J Surg 1996;83:79-82.

29. Boloker J, Bateman DA, Wung JT, Stolar CJH. Congenital diaphragmatic hernia in 120 infants treated consecutively with permissive hypercapnia/spontaneous respiration/elective repair. J Pediatr Surg 2002;37:357-66. 
30. Breaux-Jr CW, Simmons MG, Georgeson KE. Management of infants with congenital diaphragmatic hernia with ECMO. In: Zwischenberger JB, Bartlett RH. ECMO. Extracorporeal Cardiopulmonary Support in Critical Care. Extracorporeal Life Support Organization; 1995. p. 291-314.
Endereço para correspondência:

Dr. Luis Ricardo Longo dos Santos

Rua Artur Bernardes, 256 - Centro

CEP 07013-030 - Guarulhos, SP

Fone: (11) 99613013 - Fax: (11) 3069.8668

E-mail: luisrls@icr.hcnet.usp.br 